CAPÍTULO 3

\title{
APROXIMACIÓN TEÓRICA AL PERFIL PSICOGRÁFICO Y AVANCES RECIENTES DE SU APLICACIÓN EN EL CONSUMO DE ALIMENTOS
}

\author{
Hilda Helena Estrada-López ${ }^{1}$, María Auxiliadora Iglesias-Navas², \\ Marly Johana Bahamón ${ }^{3}$
}

1 Administradora de Empresas, Universidad Autónoma del Caribe. Doctora en Administración de Empresas, Universidad Autónoma de Querétaro, México. Investigadora del Grupo Innovación y Desarrolo Empresarial-GIDE. Universidad Simón Bolívar, Barranquilla (Colombia). Docente en la Facultad de Ciencias Económicas de la Universidad del Atlántico, Barranquilla (Colombia). hileslo@hotmail.co

2 Magíster en Administración de Empresas e Innovación; Ingeniera de Mercados, Universidad Simón Bolivar, Barranquilla (Colombia). miglesias1@unisimonbolivar.edu.co

3 Magíster en Educación y Desarrollo Humano, Universidad de Manizales; Psicóloga Universidad Surcolombiana de la ciudad de Neiva (Colombia).

mbahamon@unisimonbolivar.edu.co 


\section{RESUMEN}

En este capítulo se realiza una aproximación empírica al perfil psicográfico y comprende los elementos involucrados en el comportamiento del consumidor, básicos para segmentar el mercado. Se muestran las tendencias de las investigaciones sobre perfiles psicográficos en relación con los alimentos. Se trata de un estudio de revisión documental en el cual se tomaron los artículos publicados en los últimos diez años, en las bases de datos Scielo, Dialnet, Scopus, Redalyc y Sciencedirect; se utilizaron como descriptores de búsqueda: psicografía, perfil psicográfico y consumidores alimentos. En total se analizaron 44 artículos de investigación sobre el tema. Los resultados de la revisión empírica muestran la existencia de tres líneas investigativas: a) estudios sobre tendencias y detección de patrones en el consumo de alimentos; b) investigaciones centradas en las percepciones, experiencias y emociones, y c) investigaciones orientadas al análisis de valores y estilos de vida en relación con el consumo de determinados productos alimenticios. Finalmente, se reflexiona sobre las posibilidades que ofrecen el análisis y estudio de factores de orden afectivo en los estudios de perfil psicográfico.

Palabras clave: Perfil psicográfico, comportamiento consumidor, consumo alimentos, psicología del consumidor, segmentación de mercado.

\section{ABSTRACT}

A theoretical approach is made to the psychographic profile to understand the elements involved in consumer behavior and market segmentation. The following are the trends in the research on psychographic profiles in relation to food. The empirical review shows the existence of three research lines: a) studies on trends and de- 
tection of patterns in food consumption; B) research focused on perceptions, experiences and emotions; and c) research oriented to the analysis of values and lifestyles in relation to the consumption of certain foodstuffs. Finally, we reflect on the possibilities offered by the analysis and study of affective factors in psychographic profile studies.

Keywords: Psychographic profile, consumer behavior, food consumption, consumer psychology, market segmentation.

\section{INTRODUCCIÓN}

Los procesos de análisis para identificar aspectos que resulten relevantes en el comportamiento del consumidor, a menudo son complejos por la naturaleza misma del ser humano que actúa como consumidor. Así, frente a un mercado de expansión cada vez más amplio, puede ser estratégico y eficaz subdividir mercados que resultan heterogéneos, en un segmento homogéneo definido por características comunes. En coherencia con lo expuesto, "es necesario que la empresa tenga una buena comprensión del comportamiento del consumidor" (Lindon, Lendrevie, Rodríguez y Dionisio, 2000, p.105). Lo que permite establecer condiciones o características específicas, con las cuales las organizaciones, pueden detectar aspectos sensibles para la comercialización de sus productos y definir estrategias de marketing.

Toda la información sobre las características del consumidor devela factores que influyen en los hábitos de compra y de consumo, sirviendo de base para la estructuración y organización de sistemas de información, comercialización y definición de estrategias de marketing (Sergueyevna, 2013). En palabras de Kotler y Armstrong 
(2012), "entender a los consumidores es una tarea indispensable" (p.57), máxime teniendo en cuenta las características de un contexto global, en el cual el consumidor tiene acceso a un mercado cada vez más competitivo, con mayor oferta y variedad de productos que satisfacen sus necesidades. El estudio de factores intrínsecos y extrínsecos involucrados en el proceso de decisión de compra, ha adquirido mayor relevancia, convirtiéndose en un aspecto necesario para los mercados contemporáneos.

El estudio sobre las características del consumidor es un fenómeno reciente que ha surgido gracias a los avances científicos sobre el tema, y que se ha dado básicamente por el encuentro interdisciplinar entre investigadores de la Teoría Económica, la Psicología y la Sociología. Así, quienes procuran el éxito en la comercialización de sus productos, deben conocer el perfil de sus clientes para cubrir la demanda potencial identificada y evitar el fracaso en sus negocios por ignorar al consumidor como un actor dinámico (Sergueyevna, 2013).

Al respecto, Carballo (2010) refiere la necesidad de concretar el alcance y viabilidad de los perfiles, pues también advierte sobre el peligro de definir tipologías y comportamientos comunes que pueden omitir la importancia de variables particulares, o características que también aportan a entender comportamientos de consumo diferenciados.

En este aspecto, los avances en la materia proponen la segmentación del mercado como una alternativa válida en la búsqueda de elementos diferenciadores. La segmentación se identifica como el proceso mediante el cual se dividen grandes poblaciones, agru- 
pándolas por características similares y conformando nuevos subgrupos que se asemejan, permitiendo a las organizaciones dirigir sus esfuerzos de manera focalizada a ofrecer lo que el cliente busca o considera que satisface sus necesidades. Los tipos de segmentación que se reconocen con mayor frecuencia son: geográficos, demográficos, psicográficos y de comportamiento (Ciribeli y Miquelito, 2015).

Teniendo en cuenta que los inicios sobre el establecimiento de perfiles tuvieron lugar en la detección de factores geográficos y demográficos mostrando resultados divergentes, este capítulo se centra en el análisis teórico y empírico del perfil psicográfico, dados los recientes avances y las posibilidades que pueden otorgar a las organizaciones interesadas en el mercadeo de sus productos.

\section{REVISIÓN TEÓRICA DEL PERFIL PSICOGRÁFICO}

El se ha denominado perfil psicográfico a una característica importante de segmentación del mercado que involucra fundamentalmente tres variables: la personalidad, la actitud y el estilo de vida (Ciribeli y Miquelito, 2015).

La personalidad se refiere al conjunto de características individuales que posee el sujeto y que lo definen como único, lo cual se relaciona íntimamente con el desarrollo de determinados tipos de pensamientos, ideas, afectos y comportamientos; el término personalidad permite describir las características que diferencian a una persona de otra, en su forma de actuar frente a una misma situación (Lindon, 2000). En el contexto de análisis del comportamiento del consumidor, la personalidad resulta ser una variable confiable para segmentar la población de acuerdo con formas consistentes 
de actuar y que pueden relacionarse con la disposición a determinados productos, su aceptación o rechazo (Kotler y Armstrong, 2003; Yanase, 2011).

Por su parte, la actitud, considerada en principio como una tendencia a realizar determinadas acciones, producto de la interacción entre factores cognitivos y afectivos, se transformó sustancialmente cuando Allport (2005) incorporó el componente conductual a esta dinámica. Recientemente, los autores coinciden en que la actitud (Arnau, 2010; Johnson, Maio y Smith-McAllen, 2005; Montané, Jariot y Rodríguez, 2007) se trata de predisposiciones a actuar de una forma específica a partir de respuestas de carácter afectivo, cognitivo y conductual. Por otro lado, Eagly y Chaiken (2007) consideran que "la actitud es una tendencia a actuar de una manera determinada que se expresa en la evaluación a favor o en contra hacia un objeto y que está condicionada por la incidencia de los elementos cognitivos, afectivos y conductuales" (p.584). Así, la actitud frente a un producto no solo dependerá de las campañas publicitarias y estrategias de marketing que se implementen para promocionar un producto sino además, del conocimiento, las creencias, los afectos que el producto o la marca generan para disponer o no a la acción de compra.

En cuanto al estilo de vida, se considera como el patrón de comportamiento ante la sociedad que persigue los objetivos deseables. Así, se considera que el estilo de vida se relaciona directamente con las elecciones de la persona sobre lo que compra, en qué gasta su tiempo y su dinero. Las dimensiones que involucra son: las actitudes, los intereses y opiniones (Ciribeli y Miquelito, 2015). El estilo de vida se refiere a la forma de vivir de las personas, 
y se encuentra ligado a componentes motivacionales y conductuales que, a su vez, son influenciados por costumbres, hábitos, modas y valores. De esta forma el estilo de vida involucra patrones de comportamiento más o menos consistentes y constantes en el tiempo (Páez y Castaño, 2010).

En términos generales, los antecedentes del término psicográfico se remontan a Demby (citado por Limeira, 2008), quien propuso la relación entre aspectos psicológicos y demográficos para segmentar a la población, argumentando que los aspectos involucrados en la decisión de compra de determinados grupos estarían direccionados por factores psicológicos, sociológicos y antropológicos.

\section{METODOLOGÍA}

La metodología utilizada en este estudio se basó en los lineamientos propuestos por Petticrew y Roberts (2008) para la ejecución de revisiones en ciencias sociales.

La búsqueda de los artículos se hizo en las bases de datos Scielo, Redalyc, Dialnet, Science Direct y Scopus. Este proceso se hizo mediante la opción avanzada en los campos: título, resumen o palabras clave. El período de tiempo en el cual se publicaron los artículos revisados fue entre 2006 y 2016, y todas las revistas en las cuales se encontraban alojados los artículos fueron arbitradas. En español se utilizaron los descriptores de búsqueda: perfil psicográfico, psicografía, comportamiento consumidor, consumo alimentos, psicología del consumidor, segmentación de mercado. En inglés los descriptores usados fueron: psychographic profile, consumer behavior, food consumption, consumer psychology, market segmentation. 
La depuración y aplicación de criterios de inclusión se realizó teniendo en cuenta que se tratará de artículos de investigación, publicados en los últimos diez años, alojados en revistas arbitradas y que hicieran alusión a características psicográficas de consumidores de alimentos. Todos los documentos obtenidos fueron importados a una colección específica creada para la revisión.

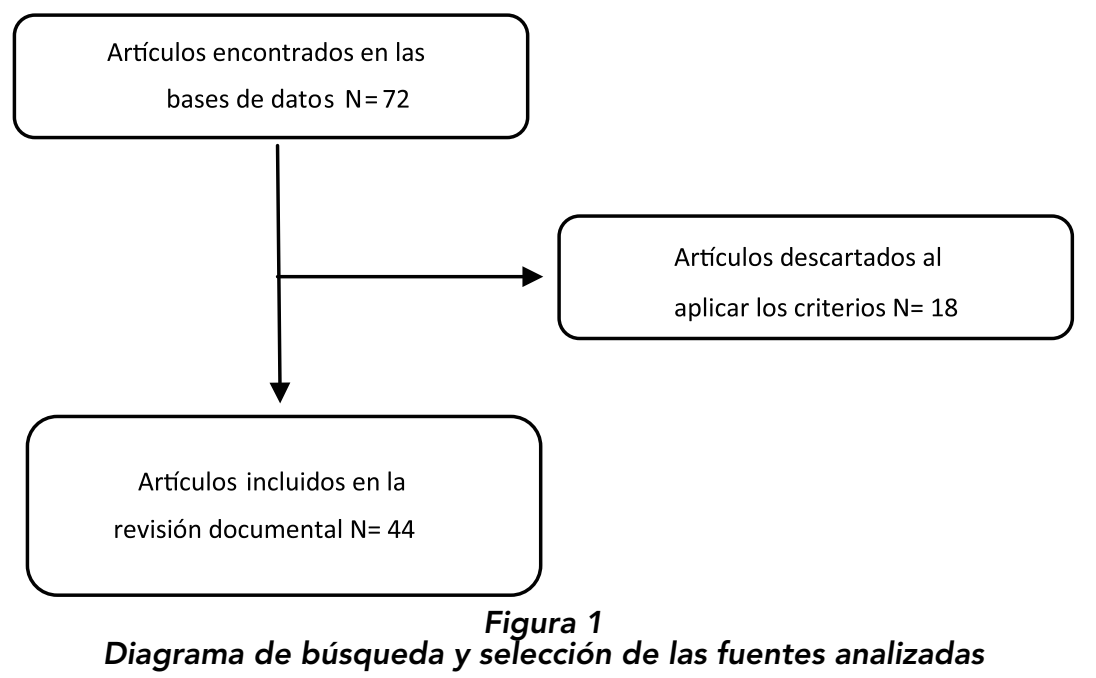

\section{Sistematización de información}

Los documentos seleccionados para la revisión fueron estudiados, y la siguiente información se extrajo a una matriz de Microsoft Excel: (a) año de publicación; (b) referencia en formato APA; (c) autor/ autores; (d) nombre de la revista; (e) título del artículo; (f) resumen; (g) país/países; (h) idioma de publicación; (i) tipo de estudio; (j) diseño metodológico; ( $k$ ) tamaño muestral; (l) estudios sobre patrones alimentarios; $(\mathrm{m})$ investigaciones relacionadas con las percepciones, experiencias y emociones en relación con los alimentos, y (n) estudios relacionados con valores, estilos de vida y consumo de alimentos. A partir de esta matriz de información se procedió a realizar el análisis de la información (Estrada-López, H. y Navia, M. 2012). 


\section{RESULTADOS Y DISCUSIÓN}

\section{Estudios recientes sobre perfiles psicográficos asociados a pa- trones de consumo de alimentos}

La comercialización de productos alimenticios puede constituirse en un nicho comercial importante, teniendo en cuenta que la demanda del consumidor es frecuente y constante. Por tanto, conocer las características de la población cautiva en torno a sus opciones de consumo y compra, se constituye en una herramienta importante para esta área. En este apartado se realizará una revisión de las investigaciones recientes en el tema y sus principales conclusiones.

Para iniciar el recorrido empírico es necesario considerar que la base teórica sobre el comportamiento y hábitos alimentarios se relaciona con los trabajos realizados por Casanueva y Morales (2001 citados por De Garine, 2002). Sobre el tema, se considera que su naturaleza resulta compleja, teniendo en cuenta que involucra factores económicos, sociales, geográficos, climáticos, además de aspectos macro, determinados por infraestructura para el transporte, las políticas agrícolas, las alimentarias, nutricionales y de salud. Individualmente también se reconocen aspectos psicológicos, familiares, culturales y religiosos (Alvarado y Luyando, 2012). En este sentido, se puede afirmar que las variables que intervienen son de diversa naturaleza, y al realizar un análisis sobre los hábitos alimentarios, será necesario considerarlos como un fenómeno multicausal que se moviliza en un ecosistema involucrando aspectos de diferente orden.

Por lo tanto, es necesario recalcar que las variables sociodemográficas han tenido un papel importante en la explicación sobre las decisiones de compra y consumo de alimentos. No obstante, 
la investigación en este campo no es suficiente para comprender en su totalidad las acciones que realizan los consumidores, por lo cual se ha optado por introducir variables psicográficas (Peterson, Hustvedt y Chen, 2008; Martínez y Montaner, 2007).

La inclusión de variables psicográficas se debe a que las variables sociodemográficas utilizadas para perfilar a los consumidores no tienen capacidad suficiente para diferenciar a las poblaciones, por lo tanto, su uso no generaría una ventaja competitiva que posibilite la homogenización de estrategias empresariales (Rivas y Grande, 2004), situación que se deriva en la consolidación de tendencias que dirigen el análisis del comportamiento del consumidor en función de sus características psicográficas, cuestión que ha favorecido el énfasis en variables como las actitudes y los valores para la segmentación de mercados (Camarena-Gómez y Sanjuán-López, 2010; Rivas y Grande, 2004).

Al respecto, los estudios y avances empíricos en el tema, muestran la existencia de varias tendencias en su estudio para entender el comportamiento del consumidor. En la revisión bibliográfica emergen tres corrientes sobre la que se fundamentan las investigaciones y que pueden denominarse de la siguiente forma: a) estudios sobre tendencias y detección de patrones en el consumo de alimentos; b) investigaciones centradas en las percepciones, experiencias y emociones, y c) investigaciones orientadas al análisis de valores y estilos de vida en relación con el consumo de determinados productos alimenticios.

\section{ESTUDIOS SOBRE TENDENCIAS Y DETECCIÓN DE PATRONES EN EL CONSUMO DE ALIMENTOS}

Teniendo en cuenta la existencia de un público cada vez mayor que busca fuentes saludables y diversas de alimentos, Brandão, Costa, 
Galizoni, Cavalcante y Neves, (2015), analizaron las características de personas que acudían a mercados locales, denominados mercados libres o de carro, para la compra de verduras y vegetales. Sus hallazgos identificaron la existencia de un perfil definido de compradores, en su mayoría mujeres, en edad de jubilación (en promedio 54 años), con interés exclusivo en comprar verduras. El precio no pareció ser un criterio de elección en esta población, como sí la ausencia de uso de pesticidas y fertilizantes químicos.

De Piero, Bassett, Rossi y Sammán (2014), desarrollaron un estudio para identificar las tendencias en el consumo de alimentos en universitarios. Los resultados encontraron que, en general, los estudiantes tienen una alimentación desequilibrada, insuficiente en cantidad y calidad de nutrientes por una selección y consumo de alimentos inadecuados. El exceso de grasa saturada y el elevado consumo de azúcares simples advierte la necesidad de promover cambios en la alimentación de los jóvenes.

Pérez-Gallardo, et al. (2015) evaluaron la ingesta de energía y nutrientes, y la adhesión a la Dieta Mediterránea en un grupo de estudiantes universitarios españoles con distinto perfil académico, y comparan la calidad de la dieta entre estudiantes que cursan grados en Ciencias de la Salud y los de otras carreras. Los encuestados, con independencia de su perfil académico, siguen una dieta hipocalórica con mayor consumo de proteínas y de grasas de las recomendadas, y menor de hidratos de carbono y fibra, lo que se refleja en el aporte insuficiente de algunas vitaminas y minerales.

Ekmeiro, J., Moreno, R., García, M. y Cámara, F. (2015), evaluaron el patrón de consumo de alimentos en familias de zonas urbanas 
de Venezuela; se estudiaron 300 grupos domésticos que aglutinaban un total de 1.163 personas. El estudio reveló que el patrón de consumo cualitativo de alimentos en la población urbana evaluada se caracteriza por prácticas alimentarias poco ajustadas a la promoción de la salud y al control de las enfermedades relacionadas con la dieta. La alimentación familiar demostró estar muy apartada de los lineamientos establecidos por las guías de alimentación para Venezuela, y que los patrones de consumo son bastante homogéneos en los diferentes estratos socioeconómicos. Los alimentos más consumidos diariamente fueron: sal, café, adobo y harina de maíz precocida; así como la carne de bovino, el pollo y las pastas alimenticias los de mayor consumo semanal. El $90 \%$ de los alimentos más consumidos diariamente son tecnológicamente procesados.

Camarena-Gómez y Sanjuán-López (2010) exploraron las preferencias hacia el origen de un alimento étnico y la influencia de variables psicográficas, teniendo en cuenta que los procesos migratorios tienen mucha importancia en sus comercializaciones. Las costumbres alimentarias se constituyen en un punto de relación con el país de origen, haciendo que se conformen segmentos de preferencias alimentarias. Investigaron las preferencias de los consumidores latinoamericanos con respecto a un alimento esencial en sus dietas, la harina de maíz. Los datos mostraron que la heterogeneidad de preferencias tuvo influencia de factores psicográficos como valores personales, la fobia a los alimentos nuevos y el etnocentrismo del consumidor. Los consumidores más neofóbicos tienden a ser más sensibles a variaciones en el precio que los más neofílicos. Los consumidores, que por sus valores personales se orientan más hacia el desarrollo personal que hacia la socialización 
y disfrute, tienden a ser personas de mayor edad y más sensibles al precio (Estrada-López, Navia, y Castañeda, 2010).

Finalmente, los consumidores con mayor grado de etnocentrismo manifiestan una preferencia más acentuada hacia la harina de su país de origen frente a la harina de otras regiones.

\section{INVESTIGACIONES CENTRADAS EN LAS PERCEPCIONES, EXPERIENCIAS Y EMOCIONES}

En esta línea, algunas investigaciones han mostrado la existencia de factores contextuales que se relacionan con las percepciones que tienen las personas frente a la comida (Meiselman, 2006, 2007; Wansink, 2004); así se ha demostrado que el contexto de la comida y la capacidad de elegir los alimentos son variables prevalentes que afectan el gusto por los alimentos (King, Meiselman, Hottenstein, Work y Cronk, 2007). En la investigación realizada por King, Meiselman y Henriques (2008), se evaluaron los efectos de la elección y la psicografía sobre la aceptabilidad de nuevos sabores, específicamente aderezos para ensaladas. Sus resultados mostraron que la posibilidad de elección tiene un efecto sobre la evaluación que el consumidor hace de los alimentos, al igual que factores como la neofobia y la neofilia.

Uijl, Jager, Graaf, WaddellyKremer (2014) realizaron una segmentación de personas mayores basada en las emociones que asocian con las comidas; la muestra se conformó por 392 adultos mayores. Los resultados permitieron identificar cuatro segmentos de consumidores: promedios placenteros, excitantes aventureros, indulgentes y restricciones indiferentes. Estos datos proporcionaron información valiosa para el desarrollo de productos y estrategias de comunicación. 
Lucchese-Cheung, Batalhay Lambert (2012) investigaron experiencias de consumo de los individuos, incluyen información correspondiente a 1.600 consumidores brasileños. Los principales resultados dan cuenta de que los alimentos impregnados de significados simbólicos, son difusores de las identidades sociales y contribuyen también con la construcción de estas identidades. La elección de alimentos por parte de los consumidores no se realiza solo en función de los aspectos sensoriales (organolépticos) de los productos, sus precios, su composición nutricional o incluso de su accesibilidad en el mercado, sino, sobre todo, en función de la representación que tienen para ellos los alimentos.

Alvarado, y Luyando, (2012) indagaron por la percepción de los adolescentes sobre el riesgo a la salud que existe en torno a su alimentación cotidiana. Uno de los principales resultados de la investigación es que los adolescentes poseen una percepción poco clara de lo que sería una alimentación saludable.

\section{INVESTIGACIONES ORIENTADAS AL ANÁLISIS DE VALORES Y ESTILOS DE VIDA}

Salgado y Camarena (2014) analizaron factores psicográficos, específicamente los valores y estilos de vida que influyen en las actitudes de los consumidores jóvenes de alimentos internacionales. La población objeto del estudio fueron consumidores jóvenes, y sus resultados muestran la existencia de dos grupos: el denominado auto-orientación (compuesto por experimentadores y hacedores), y los luchadores e innovadores; además de esto, encontraron que la influencia de las diferencias culturales es alta.

Otro estudio relevante fue desarrollado por Schnettler et al. (2012), quienes buscaron diferencias y caracterizar tipologías de consu- 
midores con sus estilos de vida relacionados con la alimentación. Sus análisis distinguieron tres tipologías principales. El grupo de personas que prefiere alimentos fáciles de preparar, planifica su alimentación y mantiene sus hábitos alimentarios (30,1 \%); los que disfrutan de salir a comer fuera y de cenas con amigos en casa, pero prefieren alimentos conocidos, son planificados y constantes en sus hábitos de alimentación (17,4\%), y los que gozan de compartir con amigos una comida, pero no les gusta cocinar ni utilizar alimentos de fácil preparación (14,6\%).

\section{CONCLUSIONES}

La segmentación de mercados a partir de variables psicográficas se ha constituido en una herramienta valiosa de análisis para entender el comportamiento del consumidor, sus hábitos de compra y consumo, y se ha incorporado con mayor fuerza en las investigaciones recientes sobre el tema para potenciar la perfilación de las poblaciones, cuestión que hasta hace poco se había concentrado en factores de orden social, económico y demográfico.

En relación con el consumo de alimentos, se evidencian tres tendencias que han guiado los estudios y que tienen en cuenta aspectos como el análisis de tendencias y patrones, la percepción, experiencias y emociones, y los valores y estilos de vida. Este análisis permite vislumbrar la existencia de otros factores de orden psicográfico, que pueden incorporarse en el estudio del comportamiento del consumidor, y que iría más allá de los clásicos estudios que involucran las actitudes, la personalidad y estilos de vida.

Las tendencias en las investigaciones ponen en evidencia la diversificación de aspectos a considerar en la comprensión del compor- 
tamiento del consumidor, y que hasta el momento dan luces sobre el papel del componente afectivo poco explorado aun.

\section{REFERENCIAS BIBLIOGRÁFICAS}

Alvarado, E. y Luyando, J. (2012). Alimentos saludables: la percepción de los jóvenes adolescentes en Monterrey, Nuevo León. Estudios sociales, 21(41), 144-164.

Allport, G. (2005). Attitudes. In C. Murchison (Ed.), Handbook of social psychology, (2), Worchester, MA: Clark University Press.

Arnau, L. (2010). La orientación de las personas desempleadas para la adaptación al cambio profesional: elaboración, aplicación y evaluación de un programa de orientación aplicado a alumnos de formación profesional para el empleo. Tesis Doctoral no publicada. Barcelona (Bellaterra): Universidad Autónoma de Barcelona.

Brandão, A., Costa, C., Galizoni, F., Cavalcante, T. y Neves, A. (2015). Perfil socioeconômico dos consumidores de hortaliças em feiras livres na microrregião de Januária. Horticultura Brasileira, 33, 119-124. doi: http://dx.doi.org/10.1590/ S0102-053620150000100019

Camarena-Gómez, D. y Sanjuán-López, A. (2010). Preferencias hacia el origen de un alimento étnico y la influencia de variables psicográficas. Economía Agraria y Recursos Naturales, 10(1), 69-97.

Carballo, D. (2010). El consumidor latinoamericano. Segmento, 48, 1-8.

Casanueva, E. y Morales, M. (2001). Nutriología médica. México: Editorial Médica Panamericana.

Ciribeli, J. y Miquelito, S. (2015). La segmentación del mercado por el criterio psicográfico: un ensayo teórico sobre los principales enfoques psicográficos y su relación con los criterios de com- 
portamiento. Revista Científica Visión de Futuro, 19(1), 33-50.

Chiang, M., Casanuevar, V., Cid, X., González, U., Olate, P., Nickel, F., y Revello, L. (2009). Factores de riesgo cardiovascular en estudiantes chilenos. Salud Pública, 41(6), 444-51.

De Garine, I. (2002). "Los aspectos socioculturales de la nutrición" en J. Contreras (ed.), Alimentación y cultura: necesidades, gustos y costumbres. México: Alfaomega-Universidad de Barcelona.

De Piero, A., Bassett, N., Rossi, A. y Sammán, N. (2014). Tendencia en el consumo de alimentos de estudiantes universitarios. Nutrición hospitalaria, 31, 1824-1831. doi: 10.3305/ nh.2015.31.4.8361

Eagly, A. y Chaiken, S. (2007). The advantages of an inclusive definition of attitude. Social Cognition, 25, 582-602.

Ekmeiro, J., Moreno, R., García, M. y Cámara, F. (2015). Patrón de consumo de alimentos a nivel familiar en zonas urbanas de Anzoátegui, Venezuela. Nutrición hospitalaria, 32, 1758-1765. doi:10.3305/nh.2015.32.4.9404

Estrada-López, H. y Navia, M. (2012). Uso de la técnica de asociación libre para conocer la percepción del consumidor sobre queso costeño en Colombia. Psicogente, 15(28). Recuperado de: http://publicaciones.unisimonbolivar.edu.co/rdigital/ojs/ index.php/psicogente/article/view/362

Estrada-López, H., Navia, M. y Castañeda, J. (2010). El ser humano como consumidor: aplicación a investigación de mercado de guayaba en el departamento del Atlántico. Psicogente, 13(24). Recuperado de: http://publicaciones.unisimonbolivar.edu.co/ rdigital/ojs/index.php/psicogente/article/view/427

Johnson, B., Maio, G. y Smith-McLallen, A. (2005). Communication and Attituo Change: Causes, Process and Effects. En Albarracín, Johnson y Zanna (Eds.), The Handbook of Attitudes. Mahwah, NJ: Lawrence Erlbaum Associates, Inc. 
King, S., Meiselman, H. y Henriques, A. (2008). The effect of choice and psychographics on the acceptability of novel flavors. Food Quality and Preference, 19, 692-696. doi:10.1016/j. foodqual.2008.05.003

King, S., Meiselman, H., Hottenstein, A., Work, T. y Cronk, V. (2007). The effects of contextual variables on food acceptability: A confirmatory study. Food Quality and Preference, 18, 58-65.

Kotler, P. y Amstrong, G. (2012). Principios de Marketing. México: Pearson Educación.

Kotler, P. y Armstrong, G. (2003). Principios de Marketing. 9. ed. São Paulo, Prentice-Hall.

Limeria, T. (2008). Comportamiento del consumidor brasilero. São Paulo: Saraiva.

Lindon, D., Lendrevie, J., Rodrigues y Dionísio, P. (2000). Mercator 2000: Teoria e Prática do Marketing. Lisboa: Publicações Dom Quixote.

Lucchese-Cheung, Th., Batalha, M. y Lambert, J. (2012). Comportamentos do consumidor de alimentos: tipologia e representação da comida. Agroalimentaria, 18(35), 115-129.

Martínez, E. y Montaner, T. (2007). "Análisis del Perfil Psicográfico de los Consumidores Propensos a las Promociones de Productos de Compra Frecuente". Cuadernos de Economía y Dirección de Empresa, 30, 063-092.

Meiselman, H. (2006). The role of context in food choice, food acceptance and food consumption. In R. Shepherd y M. Raats (Eds.), The psychology of food choice, 179-200. Waalingford: CABI.

Meiselman, H. (2007). The impact of context and environment on consumer food choice. In L. Frewer y H. van Trijp (Eds.), Understanding consumers of food products, 67-92. Cambridge: Woodhead. 
Montané, J., Jariot, M. y Rodríguez, M. (2007). Actitudes, Cambio de Actitudes y Conducción segura: Un enfoque crítico aplicado a la reducción de accidentes. Barcelona: Laertes.

Páez, M. y Castaño, J. (2010). Estilos de vida y salud en estudiantes de una facultad de Psicología. Psicología El Caribe, 25, 155-78.

Pérez-Gallardo, L., Mingo, T., Bayona, I., Ferrer, M., Márquez, E., Ramírez, R., Navas, C. y Navas, F. (2015). Calidad de la dieta en estudiantes universitarios con distinto perfil académico. Nutrición hospitalaria, 31, 2230-2239. doi: 10.3305/nh.2015.31.5.8614

Peterson, H., Hustvedt, G. y Chen, Y. (2008). "US Consumers' Willingness to Pay for Wool Product Attributes". Southern Agricultural Economics Association, Annual Meeting, Dallas, TX.

Petticrew, M. y Roberts, H. (2008). Systematic Reviews in the SocialSciences. New York, NY: John Wiley y Sons.

Rivas, J. y Grande, I. (2004). Comportamiento del Consumidor. Madrid: Esic.

Salgado, L. y Camarena, D. (2014). Los valores y estilos de vida de los jóvenes como factores de influencia en el consumo de alimentos internacionales. Poliantea, 10(19), 147-166.

Schnettler, B., Mora, M., Mills, N., Miranda, H., Sepúlveda, J., Denegri, M. y Lobos, G. (2012). Tipologías de consumidores según el estilo de vida en relación a la alimentación: Un estudio exploratorio en el sur de Chile. Revista Chilena de Nutrición, 39(4), 165-172.

Sergueyevna, N. (2013). Las características demográficas, culturales y psicográficas del consumidor centroamericano. Negotium, 9(25), 21-36.

Thurstone, L. (2001). Attitudes can be measured. American Journal of Sociology, 33, 529-554.

Uijl, L., Jager, G., Graaf, C., Waddell, J. y Kremer, S. (2014). It is not just a meal, it is an emotional experience-Asegmentation of older 
persons based on the emotions that they associate with mealtimes. Appetite, 83, 287-296. doi: http://dx.doi.org/10.1016/j. appet.2014.09.002

Wansink, B. (2004). Environmental factors that increase the food intake and consumption volume of unknowing consumers. Annual Reviews of Nutrition, 24, 455-479.

Yanaze, M. (2011). Gestão de marketing e comunicação: avanços e aplicações. 2 ed. São Paulo, Saraiva.

\section{Cómo citar este capítulo}

Estrada-López, H. H., Iglesia-Navas, M. A., \& Bahamón, M. J. (2017). Aproximación teórica al perfil psicográfico y avances recientes de su aplicación en el consumo de alimentos. En H. H. Estrada-López, H. G. Saumett-España, M. A. Iglesias-Navas, M. J. Bahamón, A. M. Cáceres-Martelo, C. E. Restrepo Flórez, . . A. Díaz Pérez, Productos de confitería nutracéutica. Una opción empresarial para cultivadores de frutas y hortalizas (pp.59-78). Barranquilla: Universidad Simón Bolívar. 